\title{
Deep Learning Based Pain Treatment
}

\author{
Tarun Jaiswal ${ }^{1}$, Sushma Jaiswal ${ }^{2}$ \\ ${ }^{1}$ MCA, Computer Science \& Information Technology, ${ }^{2}$ Computer Science \& Information Technology \\ 1,2Guru Ghasidas Central University, Bilaspur, Chhattisgarh, India
}

\begin{abstract}
How to cite this paper: Tarun Jaiswal | Sushma Jaiswal "Deep Learning Based Pain Treatment" Published in International Journal of Trend in Scientific Research and Development (ijtsrd), ISSN: 24566470, Volume-3 | Issue-4, June 2019, pp.193-211, URL: https://www.ijtsrd.c om/papers/ijtsrd23 639.pdf

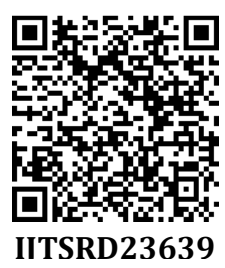

Copyright (C) 2019 by author(s) and International Journal of Trend in Scientific Research and Development Journal. This is an Open Access article distributed under the terms of the Creative Commons

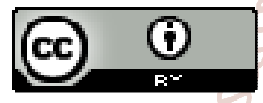
Attribution License (CC BY 4.0) (http://creativecommons.org/licenses/ by/4.0)

\section{INTRODUCTION}

In the last decade, the amount of biomedical data gathered by the bioinformatics and neuroscience communities has grown exponentially. This large amount of data, coming in different forms such as genomes, gene expression data, gene in bioinformatics, or many modalities of structural and functional imaging in neuroscience, led to the need for efficient and effective computational tools to analyze and interpret these data.

In neuroscience, neuroimaging techniques, such as computerized tomography (CT), positron emission tomography (PET), functional magnetic resonance imaging (fMRI), and diffusion tensor imaging (DTI), are used to study brains in vivo and to understand the inner workings of the nervous system. One of the main research goal of this field is to analyze the human brain network, often referred to as human connectome, in order to understand its anatomical and functional organization. Gaining such an understanding is fundamental to support early diagnoses of neurological disorders and to improve treatments of these pathologies. Indeed, connectome data analysis has led to the discovery of biomarkers associated to several neurological conditions. Neuroimaging data are complex and high dimensional and come in a wide range of spatial and temporal resolutions; for these reasons, advanced analysis techniques are necessary to describe data derived from each imaging method.

Machine learning (ML) deals with the analysis and development of models and algorithms able to learn from data in order to perform predictive analysis. Here, learning is intended as the capability of ML models to adapt their configurations to optimally explain the observed data. Usually, the objective is to extract knowledge from a data collection in order to make predictions about new, previously unseen, patterns.

\section{Machine Learning}

In this brief, we will attempt to introduce a few basic techniques that are widely applicable and then show how these can be used in various medical data imaging settings using examples from our past work in this field. For further information, interested researcher should refer well-known introductions to machine learning, such as the excellent treatments with modern technologies in the field of Pharmaceutical Research (PR).

Artificial Intelligence is a consideration of intelligence of machines, is shorthand for any task a computer can accomplish just as well as and better than, human being. But there are different forms of computer intelligence to consider when thinking about its role in Pharmaceutical research. Most of the simulated based solutions now emerging in healthcare do not rely on independent computer intelligence. Rather, they use mortal fashioned algorithms as the basis for analyzing data and recommending usages for patients. By distinction, "machine learning" trusts on neural networks. Such applications involve multilevel probabilistic analysis (MPA), allowing computers to mimic and even enlarge on the 
way the human mind processes data. As a result, not even the computer scientist can be sure how their computer programs will originate resolutions. There's yet another AI variant, known as "deep learning," wherein software learns to recognize patterns in distinctive layers. In healthcare, this mechanism is becoming increasingly useful. Because each neural-network layer operates both independently and in concert - separating aspects such as color, size and shape before integrating the outcomes - these newer digital painterly tools hold the promise of transforming diagnostic medicine and can even search for brain cancer or other type of cancers at the individual cell level. Artificial Intelligence (AI) can be sliced and diced many different ways, but the best way to understand its potential use in healthcare is to break down its applications into three separate categories: algorithmic explanations, painterly tools and medical practice in PR.

\section{Machine Learning And Biomedicine Sub-fields}

Machine Learning is a branch of AI- has been well prearranged as an effective tools for investigators, for solving investigative and extrapolative problems in a variety of medicinal arenas. ML is being used for the analysis of the importance of medical constraints and their mixtures for prediction, likewise prediction of disease progression, removal of medicinal acquaintance for consequence investigation, treatment preparation and provision, and for the complete easy-going administration. ML is also being used for data investigation, such as detection and recognition of symmetries in the data by suitably allocating with deficient data, clarification of continuous data, used in the Exhaustive Precaution Unit, and intellectual alarming resultant in effective and efficient observing. It is argued that the successful implementation of ML approaches can assistance the integration of computational schemes in the healthcare environment providing opportunities to enable and improve the work of medicinal specialists and eventually to recover the competence and superiority of medicinal healthcare. Over the past few years, there have been a number of publications of investigation outcomes that prerogative that Deep Learning (DL) has been practical to, and often times instantaneously outclasses clinicians in, a particular capacity of judgement. One of the industries currently being transformed by ML today is the healthcare production and, in this review paper, we have summarize and assembled, important applications of machine learning inside healthcare that are currently either being used or industrialized.

The One Hundred Year Study on Artificial Intelligence and machine learning is an enduring study of $\mathrm{AI} / \mathrm{ML}$ and its impact on individuals and humanity. The shallow neural network (SNN) started in 1940's. The eight relevant areas that are considered most salient include healthcare (the other seven being transference, service automata, learning, lowresource societies, community security and safety, occupation and workroom, and showbiz). Already used in areas such as radiology, pathology, genomic medicine, cardiology, outpatient services, and intensive care, AI will continue to have escalating impact in medicine although with associated fear amongst participants for an AI "takeover", especially their employments. Generally there are three Main types of ML subfields: Supervised, Unsupervised and Reinforcement Machine Learning, but in literature there are enormous types of subfields of ML available. The Figure 1 shows the major ML methods in biomedicine Sub-fields.

PubMed Central® (PMC) [68] is a free full-text documentation of biomedical and life sciences journal literature at the U.S. National Institutes of Health's National Library of Medicine. In a PubMed search for reports for artificial intelligence and medicine yielded 11,751 and almost equal number of 12,792 in surgery since 1950 . Interestingly, a search in public health resulted in 35,098 publications. Of note, there are some reports that are focused on robotics under surgery and surgical subspecialties. AI-related reports in various subspecialties since 1950 are separated into "high', "medium", and "low" use groups. The subspecialties range from almost 10,001 reports in Oncology. It is not surprising given its focus on genomic sequencing data and drug discovery, to about 6,000 in medical image-intensive fields such as Pathology and Radiology, and more than 1,000 articles in data-intensive fields (such as Epidemiology and Critical Care Medicine). Interestingly, Neurology and Neurosurgery are on this list of top subspecialties that have an $\mathrm{AI}$ presence as there is much emphasis of neuroscience currently in artificial intelligence. Finally, most subspecialties, even in low and medium use subspecialties, have seen an increase of at least 59\% in number of published reports from 2005 to 2016 . Of note, there is an incredulously low of $27 \mathrm{AI}-$ related publications in Neonatology likewise astonishing especially since it is a data-focused specialty in a critical care setting like Critical Care Medicine.

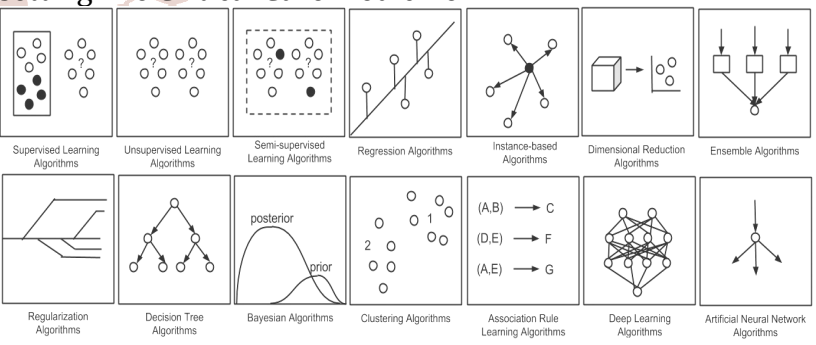

Figure 1: Major Machine Learning Methods

\begin{tabular}{|l|l|}
\hline $\begin{array}{l}\text { Supervised } \\
\text { Learning(SL). }\end{array}$ & $\begin{array}{l}\text { SL as the name designates a presence of supervisor as Instructor. Basically supervised learning } \\
\text { is a learning in which we demonstrate or train the machine using data which is well labeled that } \\
\text { means some data is already identified with correct answer. }\end{array}$ \\
\hline $\begin{array}{l}\text { Unsupervised } \\
\text { Learning(USL). }\end{array}$ & $\begin{array}{l}\text { USL is the training of machine using material that is neither categorized nor tagged and } \\
\text { allowing the algorithm to act on that information without supervision. }\end{array}$ \\
\hline $\begin{array}{l}\text { Semi-Supervised } \\
\text { Learning(SSL). }\end{array}$ & $\begin{array}{l}\text { Problems where you have a large amount of available data and only some of the data is } \\
\text { categorized, are called SSL problems. }\end{array}$ \\
\hline $\begin{array}{l}\text { Reinforcement } \\
\text { Learning(RL). }\end{array}$ & $\begin{array}{l}\text { RL is an important type of Machine Learning where an agent learn how to perform in a } \\
\text { atmosphere by execution actions and seeing the outcomes. }\end{array}$ \\
\hline $\begin{array}{l}\text { Evolutionary } \\
\text { learning(EL). }\end{array}$ & $\begin{array}{l}\text { Biological evolution can be seen as a learning process: living structure adapt to improve their } \\
\text { continuity rates and chance of having minor in their environment. We'll look at how we can } \\
\text { model this in a computer, using an idea of fitness, which corresponds to a score for how good the } \\
\text { current solution is. }\end{array}$ \\
\hline
\end{tabular}




\begin{tabular}{|l|}
\hline $\begin{array}{l}\text { Multitask Learning } \\
\text { Technique (MLT). }\end{array}$ \\
\hline
\end{tabular}

Ensemble Learning(EL).

Active learning(AL).

\begin{tabular}{|l|}
\hline Multi-label \\
learning(MLL).
\end{tabular}

Transfer learning(TL).

Transductive

inference(TI).

Genetic Algorithms and

Genetic

Programming(GA\& GP).

Rule Induction (RI).

Dictionary Learning

Technique (DL).

Representation

Learning(RL).

Distributed and parallel

learning(DAPL).

Knowledge Transfer

Learning(KTL)
MLT has a simple task of helping other learners to perform efficiently. When multitask learning algorithms are performed on a task, it remembers the procedure how it solved the problem or how it reaches to the particular conclusion.

When various individual learners are combined to form only one learner then that particular type of learning is called EL.

AL (known as "query learning" or "optimal experimental design") is a subfield of machine learning and, more generally, AI. The key hypothesis is that if the learning algorithm is allowed to choose the data from which it learns — to be "curious," if you will-it will perform better with less training.

MLL studies the problem where each example is represented by a single instance while associated with a set of labels simultaneously.

TL has been proposed to allow the domains, tasks, and circulations to be different, which can extract knowledge from one or more source tasks and apply the knowledge to a target task. The benefit of transfer learning is that it can intelligently apply knowledge learned beforehand to solve new problems faster.

As in the semi-supervised scenario, the learner receives a labeled training sample along with a set of unlabeled test points. However, the objective of TI is to predict labels only for these particular test points. However, as in the semi-supervised setting, the assumptions under which a better performance can be achieved in this setting are research questions that have not been fully resolved.

The genetic approach to ML is a relatively new concept. Both genetic algorithms (GA) and Genetic Programming (GP) are a form of evolutionary computing(EC) which is a collective name for problem solving techniques based on the principles of biological evolution like natural selection. Genetic algorithms use a vocabulary borrowed from natural genetics in that they talk about genes (or bits), chromosomes (individuals or bit strings), and population (of individuals) [1]. GA approach is centered on three main processes- crossovers, mutation and selection of individuals. Genetic algorithms are based upon the Darwin theory of "The survival of the Fittest" depending upon the fitness function the best possible solutions are selected from the pool of individuals.

RI is another very important ML method and it is easier because the rules in rule induction are transparent and easy to interpret than a regression model or a trained neural network. This model employs condition-action rules, decision trees, or similar knowledge structures. Here the performance element sorts instances down the branches of the decision tree or finds the first rule whose conditions match the instance, typically using an all-or none match process [2]. Information about classes or predictions is stored in the action sides of the rules or the leaves of the tree.

DLT, or as it is often called sparse coding, is a basic building block of many machine learning systems. This algorithmic primitive arises in applications ranging from denoising, edgedetection, super-resolution and compression. DLT is also used in the design of some DL architectures. Popular approaches to solving this problem in practice are variants of the standard alternating minimization approach.

RL aims to achieve that a reasonably sized learned representation can capture a huge number of possible input configurations, which can greatly facilitate improvements in both computational efficiency and statistical efficiency. In order to give impetus to the multi domain learning ability of representation learning, automatic representation learning, biased representation learning, cross-domain representation learning, and some other related techniques have been proposed in current years. The rapid increase in the technical activity on representation learning has been accompanied and sustained by a remarkable string of empirical successes in real-world applications, such as speech recognition, natural language processing (NLP), and intelligent vehicle systems.

DAPL seems to be a auspicious research since allocating the learning process among several workstations is a natural way of scaling up learning algorithms. Different from the traditional learning context, in which one requires the collection of that data in a database for central processing, in the framework of distributed learning, the learning is carried out in a distributed manner. In the past years, several popular distributed machine learning algorithms have been proposed, including decision rules, stacked generalization, meta-learning, and distributed boosting. With the advantage of distributed computing for managing big volumes of data, distributed learning avoids the necessity of gathering data into a single workstation for central processing, saving time and energy [3].

According to Soumava Roy et.al. [4] KTL Techniques that benefit from available knowledge to work around a difficult learning problem (e.g., learning tasks with insufficient data for training) are commonly referred to as knowledge transfer methods. In this part, we first discuss the problem of Zero- and One-Shot Learning (ZSL/OSL) from deep learning viewpoint. As the names imply, the number of training samples per class is zero for ZSL and merely one for OSL.

Finally, we examine Teacher-Student Networks (TSNs) [5] that distil the knowledge of a large 


\begin{tabular}{|c|c|}
\hline & $\begin{array}{l}\text { model into a smaller one. } \\
\text { A. } \quad \text { Zero- and One-Shot Learning (ZSL/OSL). } \\
\text { B. } \quad \text { Zero-Shot Learning (ZSL). } \\
\text { C. } \quad \text { One-Shot Learning (OSL). } \\
\text { D. } \quad \text { Teacher-Student Networks Learning (TSNL). } \\
\text { E. } \quad \text { Few-Shot Learning (FSL). }\end{array}$ \\
\hline Batch learning(BL). & $\begin{array}{l}\text { In BL, the system is incapable of learning incrementally: it must be trained using all the available } \\
\text { data. This is called offline learning. If you want a BL system to know about new data (such as a } \\
\text { new type of spam), you need to train a new version of the system from scratch on the full dataset } \\
\text { (not just the new data, but also the old data), then stop the old system and replace it with the } \\
\text { new one. }\end{array}$ \\
\hline Online learning (OL). & $\begin{array}{l}\text { In } \mathrm{OL} \text {, we train the system incrementally by feeding it data instances sequentially, either } \\
\text { individually or by small groups called mini-batches. OL is great for systems that receive data as a } \\
\text { continuous flow (e.g., stock prices) and need to adapt to change rapidly or autonomously. }\end{array}$ \\
\hline $\begin{array}{l}\text { Decision tree based } \\
\text { algorithms (DTBA). }\end{array}$ & $\begin{array}{l}\text { DTBA define models that are iteratively or recursively constructed based on the data provided. } \\
\text { The goal of Decision tree based algorithms is to predict the value of a target variable given a set } \\
\text { of input variables. Decisions fork in tree structures until a prediction decision is made for a given } \\
\text { record. }\end{array}$ \\
\hline $\begin{array}{l}\text { Bayesian method based } \\
\text { algorithms(BMBA). }\end{array}$ & $\begin{array}{l}\text { BMBA are those that explicitly apply the Bayesian inference theorem and again solve } \\
\text { classification and regression problems. Bayesian methods facilitate subjective probability in } \\
\text { modeling. }\end{array}$ \\
\hline $\begin{array}{l}\text { Kernel method based } \\
\text { algorithms(KMBA). }\end{array}$ & $\begin{array}{l}\text { When we hear about kernel methods, the first thing that comes to mind is Support Vector } \\
\text { Machines (SVM). These methods are usually a group of methods in themselves. Kernel methods } \\
\text { are concerned with configurations analysis and as explained in the preceding sections, that crux } \\
\text { of configurations analysis includes various mapping techniques. }\end{array}$ \\
\hline Clustering Methods(CM). & $\begin{array}{l}\text { Clustering, like regression, describes a class of problems and a class of methods. Clustering } \\
\text { methods are typically organized by the modeling approaches such as centroid-based and } \\
\text { hierarchical. }\end{array}$ \\
\hline $\begin{array}{l}\text { Artificial Neural } \\
\text { Networks (ANN). }\end{array}$ & $\begin{array}{l}\text { Similar to kernel methods, ANN are again a class of configurations matching techniques, but } \\
\text { these models are inspired by the structure of biological neural networks. These methods are } \\
\text { again used to solve classifications and regression problems. They relate to DL modeling and have } \\
\text { many subfields of algorithms that help solve specific problems in context. }\end{array}$ \\
\hline $\begin{array}{l}\text { Dimensionality } \\
\text { Reduction(DR). }\end{array}$ & $\begin{array}{l}\text { Like clustering methods, DR methods work iteratively and on the data structure in an } \\
\text { unsupervised manner. Given the dataset and the dimensions, more dimensions would mean } \\
\text { more work in the Machine learning implementation. The idea is to iteratively reduce the } \\
\text { dimensions and bring more relevant dimensions forward. }\end{array}$ \\
\hline $\begin{array}{l}\text { Instance based learning } \\
\text { algorithms(IBLA). }\end{array}$ & $\begin{array}{l}\text { Instances are nothing but subsets of datasets, and IBLA work on an identified instance or groups } \\
\text { of instances that are critical to the problem. The results across instances are compared, which } \\
\text { can include an instance of new data as well. This comparison uses a particular similarity measure } \\
\text { to find the best match and predict. Instance based methods are also called case-based or } \\
\text { memory-based learning. Here the focus is on the representation of the instances and similarity } \\
\text { measures for comparison between instances. }\end{array}$ \\
\hline $\begin{array}{l}\text { Regression analysis } \\
\text { based algorithms } \\
\text { (RABA). }\end{array}$ & $\begin{array}{l}\text { Regression is a process of refining the model iteratively based on the error generated by the } \\
\text { model. Regression also is used to define a ML problem type. }\end{array}$ \\
\hline $\begin{array}{l}\text { Association rule based } \\
\text { learning algorithms } \\
\text { (ARBLA). }\end{array}$ & $\begin{array}{l}\text { Given the variables, association rule based learning algorithms extract and define rules that can } \\
\text { be applied on a dataset and demonstrate experienced-based learning, and thus prediction. These } \\
\text { rules when associated in a multi-dimensional data context can be useful in a commercial context } \\
\text { as well. }\end{array}$ \\
\hline Fuzzy Logic (FL). & $\begin{array}{l}\text { FL was first industrialized by Zadeh [6] in the mid-1960s for representing uncertain and vague } \\
\text { knowledge. It provides an estimated but effective means of describing the behavior of systems } \\
\text { that are too complex, ill-defined, or not easily analyzed precisely. Fuzzy variables are handled } \\
\text { using a system called a fuzzy logic controller. It includes fuzzification, fuzzy inference, and } \\
\text { defuzzification. Zadeh discusses that the attempts to automate various types of activities from } \\
\text { assembling hardware to medical diagnosis have been impeded by the gap between the way } \\
\text { human beings reason and the way computers are programmed. FL uses graded statements } \\
\text { rather than ones that are strictly true or false. }\end{array}$ \\
\hline Exact learning (EL). & $\begin{array}{l}\text { In this setting the goal is to learn a target concept from the ability to interact with it. For } \\
\text { concreteness, we focus on learning target concepts that are Boolean functions: the target is some } \\
\text { unknown } \mathrm{c}:[0,1\}^{n} \rightarrow[0,1] \text { coming from a known concept class } C \text { of functions, [7] and our goal is } \\
\text { to identify } c \text { exactly, with high probability, using membership queries (which allow the learner to } \\
\text { learn } ₫(x) \text { for } x \text { of his choice). }\end{array}$ \\
\hline
\end{tabular}




\begin{tabular}{|c|c|}
\hline $\begin{array}{l}\text { PAC (Probably } \\
\text { Approximately Correct) } \\
\text { learning. }\end{array}$ & $\begin{array}{l}\text { In this setting one also wants to learn an unknown } c:\{0,1\}^{\mathrm{n}} \rightarrow\{0,1\} \text { from a known concept class } \\
\mathrm{c} \text {, but in a more passive way than with membership queries: the learner receives several labeled } \\
\text { examples }(\mathrm{x}, \mathrm{c}(\mathrm{x})) \text {, where } \mathrm{x} \text { is distributed according to some unknown probability distribution } \mathbb{D} \\
\text { over }\{0,1\}^{\mathrm{n}} \text {. The learner gets multiple i.i.d. labeled examples. From this limited "view" on } \mathrm{c} \text {, the } \\
\text { learner wants to generalize, producing a hypothesis h that probably agrees with c on "most" } \mathrm{x} \text {, } \\
\text { measured according to the same } \mathrm{D} \text {. This is the classical PAC model. }\end{array}$ \\
\hline Agnostic learning(AL). & $\begin{array}{l}\text { In this setting one wants to approximate a distribution on }\{0,1\}^{\mu+1} \text { by finding a good hypothesis } \\
\mathrm{h} \text { to predict the last bit from the first } \mathrm{n} \text { bits. A "good" hypothesis is one that is not much worse } \\
\text { than the best predictor available in a given class } \mathcal{C} \text { of available hypotheses. The agnostic model } \\
\text { has more freedom than the PAC model and allows to model more realistic situations, for example } \\
\text { when the data is noisy or when no "perfect" target concept exists. Like in the PAC model, it turns } \\
\text { out quantum sample complexity is not significantly smaller than classical sample complexity in } \\
\text { the agnostic model [7]. }\end{array}$ \\
\hline $\begin{array}{l}\text { Metric Learning Method } \\
\text { (MLM). }\end{array}$ & $\begin{array}{l}\text { This approach involves learning a metric space in which learning is particularly efficient. This } \\
\text { approach has mostly been used for few-shot classification. Intuitively, if our goal is to learn from } \\
\text { a small number of example images, than a simple approach is to compare the image that we are } \\
\text { trying to classify with the example images that you have. But, as we might imagine, comparing } \\
\text { images in pixel space won't work well. Instead, we can train a Siamese network or perform } \\
\text { comparisons in a learned metric space. }\end{array}$ \\
\hline $\begin{array}{l}\text { Classical machine } \\
\text { learning (CML). }\end{array}$ & $\begin{array}{l}\text { CML and data analysis can be divided into several categories. First, computers can be used to } \\
\text { perform 'classic' data analysis methods such as least squares regression, polynomials } \\
\text { interpolation, and data analysis. Machine learning protocols can be supervised or unsupervised. }\end{array}$ \\
\hline $\begin{array}{l}\text { Quantum Machine } \\
\text { Learning(QML). }\end{array}$ & $\begin{array}{l}\text { According to Cristian Romero Garcia [8] QML aims to implement machine learning algorithms in } \\
\text { quantum systems, by using the quantum properties such as superposition and entanglement to } \\
\text { solve these problems efficiently. The field of classical machine learning is receiving lot of } \\
\text { attention and investments from the industry. Nowadays, due to the huge quantities of data with } \\
\text { which we deal every day, new approaches are needed to automatically manage, organize and } \\
\text { classify these data. Classical machine learning, which is a flexible and adaptable procedure, can } \\
\text { recognize configurations efficiently, but some of these problems cannot be efficiently solved by } \\
\text { these algorithms. The companies whose labour consists in big databases managementare aware } \\
\text { of these limitations, and are very interested in new approaches to accomplish this. They have } \\
\text { found in quantum machine learning one of these approaches. Nevertheless, there is not a general } \\
\text { theory to analyze and engineer new QML algorithms, and there are additionally some } \\
\text { unanswered related questions. } 456-6470\end{array}$ \\
\hline $\begin{array}{l}\text { Adversarial Machine } \\
\text { Learning(AML). }\end{array}$ & $\begin{array}{l}\text { Systems using ML have been successfully deployed for fighting spam, fraud, and other malicious } \\
\text { activities. These systems typically consist of a classifier that flags certain instances as malicious } \\
\text { based on a fixed set of features. For example, spam filters classify each incoming email message } \\
\text { as spam or legitimate email by using a set of features such as which words are present. } \\
\text { Unfortunately, as classifiers become more widely deployed, the incentive for defeating them } \\
\text { increases. }\end{array}$ \\
\hline
\end{tabular}

Temporal difference learning (TDL).

Multi-Task Learning (MTL).
TDL IS AN APPROACH TO LEARNING HOW TO PREDICT A QUANTITY THAT DEPENDS ON FUTURE VALUES OF A GIVEN SIGNAL. THE NAME TDL DERIVES FROM ITS USE OF CHANGES, OR DIFFERENCES, IN PREDICTIONS OVERSUCCESSIVE TIME STEPS TO DRIVE THE LEARNING PROCESS.

Multi-task learning is an approach used to aggregate together similar tasks or problems and train a computer system to learn how to resolve collectively the tasks or problems. This leads to the resolution of more complex tasks or problems within the shortest possible time using machine learning.

Greedy Learning Algorithms (GLA).
We will look into a common computer science algorithm technique called the greedy algorithms The idea of greedy algorithm is to make each choice in a locally optimal manner.
Cloud Machine Learning (CML).
The commercial clouds are in a race to see who can provide the most interesting and useful AI services on their cloud platform. The big breakthroughs came when deep learning models trained on massive data collections began to reach levels of human accuracy. 
Meta-learning System (MLS).

\section{Q-Learning (QL).}

Fog Computing (FC).

Edge Computing (EC).

Socially Guided Machine Learning (SGML). learning (ROBOTML).

The Hebbian Learning(HL).

\section{Competitive}

Learning(CL).

The Boltzmann learning (BL).
MLS are trained by being exposed to a large number of tasks and are then tested in their ability to learn new tasks; an example of a task might be classifying a new image within 5 possible classes, given one example of each class, or learning to efficiently navigate a new maze with only one traversal through the maze. During MLS, The Model Is Trained To Learn Tasks In The MetaTraining Set. There Are Two Optimizations At Play - The Learner, Which Learns New Tasks, And The Meta-Learner, Which Trains The Learner. Methods For Meta-Learning Have Typically Fallen Into One Of Three Categories: Recurrent Models, Metric Learning, And Learning Optimizers[9]. QL [10], is widely used in Reinforcement Learning (RL) and has had many positive results in playing stochastic games, elevator control and robotics. Q-Learning produces an action value function or $Q$ function, $Q: S \times A \rightarrow \mathbb{R}$, which maps every state-action pair to the expected utility of taking the action in the state and following the greedy policy thereafter. The greedy policy of a $\mathrm{Q}$ function is one that simply chooses the action with the highest Q-value for any state. QL is an offpolicy learning algorithm, meaning it can learn from example trajectories that differ from its policy. To learn from demonstrations, we take advantage of this ability and simply perform QL along the demonstrated trajectory.

According to M.S. Mahdavinejad et.al.[11], the architecture of fog computing is applied to migrate information from the data centers task to the edge of the servers. This architectures is built based on the edge servers.This architecture has been and is being implemented in vital areas like eHealth and military applications.

According to M.S. Mahdavinejad et.al. [11],In this architecture, processing is run at a distance from the core, toward the edge of the network. This type of processing enables data to be initially processed at the edge devices. Devices at the edge may not be connected to the network in a continuous manner, so they need a copy of master data/reference data for online processing. Edge devices have different features such as 1) enhancing security, 2) filtering and cleaning of the data, and 3) storing local data for local use.

Agents (e.g., robotic helpers, non-player characters in games, etc.) that operate in human environments will be more flexible if they are able to learn new tasks and skills from their endusers. We recognize that while the average person is not familiar with machine learning techniques, they are intimately familiar with social learning (e.g. Tutelage, imitation). SGML [12] explores ways in which machine learning can be designed to more fully take advantage of natural human interaction and tutelage. It asks questions like "how do people want to teach agents?" and "how do we design agents to learn effectively from natural human interaction and instruction?" Inspiration for SGML comes (in part) from Situated Learning Theory, a field of study that looks at the social world of children and how it contributes to their development. A key concept is scaffolding, where a teacher provides support such that a learner can achieve something they would not be able to accomplish independently.

Considering the structure of a typical robot system, there are numerous opportunities to learn. They include learning of direct sensor-actor couplings on the lowest level of robot control as well as the acquisition of knowledge about the robot's environment or the continuous improvement of the robot's reasoning and planning capabilities, in order to enable it to handle increasingly complex tasks over time.

Donald Hebb is credited with being among the first to think about this problem and attempt to come up with a plausible solution in his 1949 book The Organization of Behavior. However, Hebb was primarily a psychologist and his ideas were stated in rather vague terms, such as: "When an axon of cell A is near enough to excite cell B and repeatedly or persistently takes part in firing it, some growth process or metabolic change takes place in one or both cells such that A's efficiency, as one of the cells firing B, is increased" often paraphrased as "Neurons that fire together wire together". Not a single equation can be found in his book. While the concept of HL has played an important role in the development of both neuroscience and ML[13].

In CL, as the name implies, the output neurons of a neural network compete among themselves for being the one to be active (fired). Thus, whereas in a neural network based on Hebbian learning several output neurons may be active simultaneously, in the case of CL a single outputneuron is active at any one time. It is this feature that makes CL highly suited to discover those statistically salient features that may be used to classify a set of input configurations[14].

The BL rule, named in honor of L. Boltzmann, is a stochastic learning algorithm derived from information-theoretic and thermodynamic considerations. Here we merely emphasize the essence of what BL is. In a Boltzmann machine, the neurons constitute a recurrent structure, and they operate in a binary manner in that they are either in an "on" state denoted by +1 or in an "off" state denoted by - 1 . The machine is characterized by an energy function E, the value of which is determined by the particular states occupied by the individual neurons of the machine[14]. 


\begin{tabular}{|c|c|}
\hline $\begin{array}{l}\text { Error-Correction } \\
\text { Learning (ECL). }\end{array}$ & $\begin{array}{l}\text { Let } T_{k}(n) \text { denote some desired response or target response for neuron } k \text { at time } n \text {. The response } \\
S_{k}(n) \text { is produced by a stimulus (vector) } x(n) \text { applied to the input of the network in which } \\
\text { neuron } k \text { is embedded. The input vector } x(n) \text { and desired response } T_{k}(n) \text { for neuron } k \text { constitute } \\
\text { a particular example presented to the network at time } n \text {. It is assumed that this example and all } \\
\text { other examples presented to the network are generated by an environment that is probabilistic } \\
\text { in nature, but the underlying probability distribution is unknown. Typically, the actual response } \\
S_{k}(n) \text { of neuron } k \text { is different from the desired response } T_{k}(n) \text {. Hence, we may define an error } \\
\text { signal as the difference between the target response } T_{k}(n) \text { and the actual response } S_{k}(n) \text {, as } \\
\text { shown by } K_{k n}(n)=T_{k}(n)-S_{k}(n) \text { The ultimate purpose of ECL is to minimize a cost function based } \\
\text { on the error signal } \mathbb{K}_{k}(n) \text {, such that the actual response of each output neuron in the network } \\
\text { approaches the target response for that neuron in some statistical sense[14]. }\end{array}$ \\
\hline Deep learning (DL). & $\begin{array}{l}\text { DL is an area of Machine learning that focuses on unifying Machine learning with artificial } \\
\text { intelligence. In terms of the relationship with artificial neural networks, this field is more of an } \\
\text { advancement to artificial neural networks that work on large amounts of common data to derive } \\
\text { practical insights. It deals with building more complex neural networks to solve problems } \\
\text { classified under semi-supervised learning and operates on datasets that have little labeled data. }\end{array}$ \\
\hline $\begin{array}{l}\text { Convolutional neural } \\
\text { networks(CNN) }\end{array}$ & $\begin{array}{l}\text { CNN are deep artificial neural networks majorly used to classify images, clustering based on } \\
\text { similarity, and object recognition. Related algorithms used to identify faces, street signs, } \\
\text { tumours, platypuses and different aspects of visual data[15]. }\end{array}$ \\
\hline $\begin{array}{l}\text { Recurrent neural } \\
\text { network (RNN) }\end{array}$ & $\begin{array}{l}\text { RNN is an advanced artificial neural network to build on earlier types of networks with fixed- } \\
\text { size input vectors and output vectors[16].The RNN is related to deep learning to simulate the } \\
\text { neural activity in the human brain. RNNs widely used for Sentiment Classification, Image } \\
\text { captioning and language translation[17]. }\end{array}$ \\
\hline
\end{tabular}

Table 1: Machine Learning And Biomedicine Sub-Fileds Details

\begin{tabular}{|c|c|c|}
\hline S. No. & Area & Details \\
\hline 1. & Evidence Based Medicine (EBM) & $\begin{array}{l}\text { It is an approach taken by medical consultants to diagnose and } \\
\text { medicate sicknesses using medical evidence. It involves } \\
\text { collection, interpretation, and summarization of evidences } \\
\text { followed by systematic retrieval of the best evidence available } \\
\text { and then applying them in practice }\end{array}$ \\
\hline 2. & Adverse Drug Events (ADE) & $\begin{array}{l}\text { It is a damage experienced by a patient resulting from the use of } \\
\text { a drug. It may occur due to suppository errors like overdose, } \\
\text { wrong medication, adverse drug reactions or wrong patient. } \\
\text { Identification of factors relating to adverse drug events is one of } \\
\text { the major areas of research in medicine. }\end{array}$ \\
\hline 3. & Healthcare Management (HM) & $\begin{array}{l}\text { It is health management using monitoring equipment and } \\
\text { devices whose objective is to help patients manage their medical } \\
\text { conditions at home. Systems are designed which help to identify } \\
\text { chronic disease state and keep track of high risk patients, and } \\
\text { design appropriate interventions and reduce number of hospital } \\
\text { admissions. }\end{array}$ \\
\hline 4. & $\begin{array}{c}\text { Predictive Data Mining (PDM) in clinical } \\
\text { medicine }\end{array}$ & $\begin{array}{l}\text { Uses enduring specific information to derive models that can } \\
\text { predict the outcome of interest and thus support clinical } \\
\text { decision making }\end{array}$ \\
\hline 5. & Medical Decision Support System (MDSS) & $\begin{array}{l}\text { It is a framework which enables Medical decision making in the } \\
\text { presence of partial information. A medical decision support } \\
\text { system is a health information technology system designed to } \\
\text { provide doctors and health professionals with assistance during } \\
\text { decision making. }\end{array}$ \\
\hline 6. & $\begin{array}{l}\text { Passive In-Home Health and Wellness } \\
\text { Monitoring }\end{array}$ & $\begin{array}{l}\text { These systems are used for monitoring older adults passively in } \\
\text { their own living settings through placing sensors in their living } \\
\text { environment. Their routine activities are analyzed and mined to } \\
\text { detect changes in their health conditions or indicators of early } \\
\text { onset of disease. }\end{array}$ \\
\hline 7. & $\begin{array}{l}\text { MARTDIAB :A Communication and } \\
\text { Information Technology (IT) Approach for } \\
\text { the Intelligent monitoring, Management } \\
\text { and Follow-Up of Type -1 Diabetes Patients }\end{array}$ & $\begin{array}{l}\text { An automated system for monitoring and management of type } 1 \\
\text { Diabetic patients is developed which supports monitoring, } \\
\text { management and treatment of patients with type } 1 \text { diabetes. }\end{array}$ \\
\hline 8. & $\begin{array}{l}\text { HCloud : A Innovative Application Oriented } \\
\text { Cloud Platform for Precautionary } \\
\text { Healthcare }\end{array}$ & $\begin{array}{l}\text { HCloud - A healthcare system is developed for preventive } \\
\text { healthcare service. It is used by many healthcare workers to } \\
\text { access and consolidate all patient electronic medical records } \\
\text { which gives fast access to patient }\end{array}$ \\
\hline
\end{tabular}


International Journal of Trend in Scientific Research and Development (IJTSRD) @ www.ijtsrd.com eISSN: 2456-6470

\begin{tabular}{|c|c|c|}
\hline 9. & Effective Management of Hospital resource & $\begin{array}{l}\text { Using data mining, it is possible to detect the chronic disease } \\
\text { and based on the complication of the patient disease prioritize } \\
\text { the patients so that they will get effective treatment in timely } \\
\text { and accurate manner. Fitness report and demographic details of } \\
\text { patients is also useful for utilizing the available hospital } \\
\text { resources effectively. }\end{array}$ \\
\hline 10. & Hospital Ranking (HR) & $\begin{array}{l}\text { Different data mining approaches are used to analyze the } \\
\text { various hospital details in order to determine their ranks. }\end{array}$ \\
\hline 11. & $\begin{array}{l}\text { Better Customer Relation } \\
\text { (BCR) }\end{array}$ & $\begin{array}{l}\text { Data Mining helps the healthcare institute to understand the } \\
\text { needs, preferences, behavior, patterns and quality of their } \\
\text { customer in order to make better relation with them. Using Data } \\
\text { Mining, Customer Potential Management Corp. develops an } \\
\text { index represent the utilization of Consumer healthcare. This } \\
\text { index helps to detect the influence of customer towards } \\
\text { particular healthcare service. }\end{array}$ \\
\hline 12. & Hospital Infection Control (HIC) & $\begin{array}{l}\text { A system for inspection is constructed using data mining } \\
\text { techniques to discover unknown or irregular patterns in the } \\
\text { infection control data. To control the infection in the hospitals, } \\
\text { this information is reviewed further by an Expert. }\end{array}$ \\
\hline 13. & Smarter Treatment Techniques (STT) & $\begin{array}{l}\text { Using Data Mining, physicians and patients can easily compare } \\
\text { among different treatments technique. }\end{array}$ \\
\hline 14. & Improved Patient & $\begin{array}{l}\text { Large amount of data is collected with the advancement in } \\
\text { electronic health record. Patient data which is available in } \\
\text { digitized form improve the healthcare system quality. In order } \\
\text { to analyze this massive data, a predictive model is constructed } \\
\text { using data mining that discover interesting information from } \\
\text { this huge data and make decision regarding the improvement of } \\
\text { healthcare quality. }\end{array}$ \\
\hline 15. & Decrease Insurance Fraud (DIF) & $\begin{array}{l}\text { Healthcare insurer develops a model to detect the fraud and } \\
\text { abuse in the medical claims using data mining techniques. Check } \\
\text { improper medicaments, false data claimed by peoples. }\end{array}$ \\
\hline 16. & Recognize High-Risk Patients (RHRP) & $\begin{array}{l}\text { The main concern of this system is to handle the diabetic } \\
\text { patients, improve their health quality and also offers cost } \\
\text { savings services to the patient. Using Predictive model, } \\
\text { healthcare provider recognize the patient which require more } \\
\text { concern as compare to other patients }\end{array}$ \\
\hline 17. & Health Policy Planning (HP) & $\begin{array}{l}\text { Data mining play an important role for making effective policy } \\
\text { of healthcare in order to improve the health quality as well as } \\
\text { reducing the cost for health services. }\end{array}$ \\
\hline 18. & Understanding Medical Data (UMD) & $\begin{array}{l}\text { In pharmaceutical research, the Internet of Things (IoT) devices } \\
\text { such as the Fitbit on the growth, the number of ways in which to } \\
\text { collect vast amounts of medical data from anonymous sources is } \\
\text { increasing. }\end{array}$ \\
\hline 19. & Robotic Surgery (RS) & $\begin{array}{l}\text { Robotic Surgery is nothing new but the recent advancements } \\
\text { already in progressive adopted in the field of PR. }\end{array}$ \\
\hline 20. & Automatic & $\begin{array}{l}\text { ML systems within robotic surgeons are one way in which } \\
\text { automation could find itself integrated into pharmaceutical } \\
\text { Research of the future. }\end{array}$ \\
\hline 21. & Radioactivity Treatments & $\begin{array}{l}\text { Robotic surgeon successfully treated as and compare healthy } \\
\text { and cancerous tissues and cells is key to then building the most } \\
\text { appropriate treatment plans whereas, the human based or } \\
\text { involvement treatment may be very risky based on radiation. }\end{array}$ \\
\hline 22. & Generating Electronic Smart Card (GESC) & $\begin{array}{l}\text { With the enormous capacities of medical and healthcare data } \\
\text { now available, the implementation of smart electronic } \\
\text { healthcare records has become essential. This, however, creates } \\
\text { even more challenges for those already working within the } \\
\text { business industry. Machine learning projects make an up a } \\
\text { significant percentage of the efforts going into assisting in the } \\
\text { creation of such smart records with handwriting recognition } \\
\text { technologies and vision of such developments. Other machine } \\
\text { learning applications in the creation of electronic smart records } \\
\text { involve using records with built-in artificial intelligence so as to } \\
\text { assist with keeping medical records, interpreting health } \\
\text { conditions and suggesting treatment plans. }\end{array}$ \\
\hline
\end{tabular}


International Journal of Trend in Scientific Research and Development (IJTSRD) @ www.ijtsrd.com eISSN: 2456-6470

\begin{tabular}{|l|l|l|}
\hline 23. & Optimizing Clinical Trials (OCT) & $\begin{array}{l}\text { There have been several calls for machine learning technologies } \\
\text { to be more closely involved in clinical research trials. }\end{array}$ \\
\hline & & $\begin{array}{l}\text { PM and treatments have been discussed and debated for many } \\
\text { years now, however, with technological advances in healthcare } \\
\text { devices, artificial intelligence, machine learning and centralized } \\
\text { system such treatments could as soon as available. As previously } \\
\text { mentioned, machine learning technologies can be used to } \\
\text { interpret the high volumes of patient data collected by IoT and } \\
\text { healthcare devices and then use these interpretations to predict } \\
\text { conditions or suggest treatments. With both the widespread } \\
\text { adoption of AI and machine learning and the increasing } \\
\text { prevalence of data-gathering medical devices, such solutions are } \\
\text { currently under investigation by industry players such as IBM } \\
\text { and could soon be commonplace within healthcare institutions } \\
\text { and facilities. }\end{array}$ \\
\hline Personalization Medicines (PM) \\
\end{tabular}

Table 2: Research Application In Healthcare

V. Medical Application last 03 years

\begin{tabular}{|c|c|c|c|c|c|c|c|}
\hline 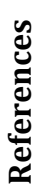 & 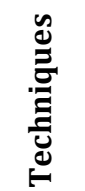 & 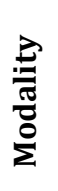 & 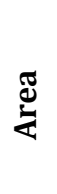 & $\underset{\succsim}{\varpi}$ & $\stackrel{\varphi}{0}$ & & Result \\
\hline$\stackrel{\infty}{=}$ & Z & $\begin{array}{l}\frac{1}{\not} \\
\stackrel{I}{I}\end{array}$ & $\frac{\nwarrow}{z}$ & 竝 & $\frac{2}{3}$ & 莡 & $\begin{array}{l}\text { This paper used multiple instance learning framework in } \\
\text { classification training with deep learning features. In this paper } \\
\text { Several interesting conclusions can be obtain : automatic feature } \\
\text { learning outperforms manual feature; the unsupervised approach } \\
\text { can achieve performance that's close to fully supervised approach } \\
93.56 \% \text { vs. } 94.52 \% \text {; and the MIL performance of coarse label 96.30\% } \\
\text { outweighs the supervised performance of fine label } 95.40 \% \text { in } \\
\text { supervised deep learning features. }\end{array}$ \\
\hline 戸 & $\sum_{\substack{1 \\
\sum}}^{1}$ & $\begin{array}{l}\frac{1}{\not} \\
\underset{I}{I}\end{array}$ & 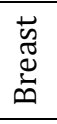 & $\begin{array}{l}0 \\
\stackrel{\circ}{\circ}\end{array}$ & 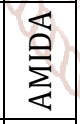 & 艺 & $\begin{array}{l}\text { The AggNet model almost performs around } 8.6 \% \text { of AUC as good as } \\
\text { the augmented ground truth model AM-GT and easily outperforms } \\
\text { both AM-MV and AM-GLAD around } 7.6 \% \text { of AUC. }\end{array}$ \\
\hline 언 & Z & $\begin{array}{l}\stackrel{1}{\not} \\
\underset{I}{\mid c}\end{array}$ & $\frac{\nwarrow}{z}$ & 공 & 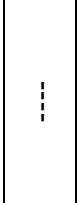 & & $\begin{array}{l}\text { Extensive experiments using the } 2015 \text { MICCAI Gland Challenge } \\
\text { dataset and a lymph node ultrasound image segmentation dataset } \\
\text { show that, using annotation suggestions by our method, state-of-the- } \\
\text { art segmentation performance can be achieved by using only } 50 \% \text { of } \\
\text { training data. }\end{array}$ \\
\hline $\bar{\sim}$ & ¿ Z & 它 & $\stackrel{0}{\Xi}$ & $\stackrel{\text { L }}{\underset{D}{0}}$ & 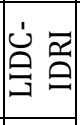 & \multirow{4}{*}{ 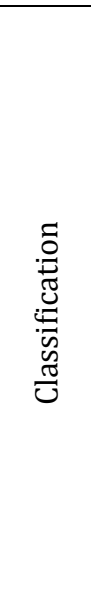 } & $\begin{array}{l}\text { Experimental results demonstrate the effectiveness of M-CNN } \\
\text { method on classifying malignant and benign nodules without nodule } \\
\text { segmentation. }\end{array}$ \\
\hline$\sqrt[N]{N}$ & $\begin{array}{l}Z \\
\text { Z } \\
\dot{m}\end{array}$ & $\vec{z}$ & 莞 & 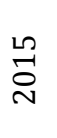 & 文 & & $\begin{array}{l}\text { Demonstrate that } 3 \mathrm{D} \text { convolutional neural networks outperform } \\
\text { several other classifiers reported in the literature and produce state- } \\
\text { of-art results. }\end{array}$ \\
\hline ָ̃ & $\begin{array}{l}Z \\
z_{1} \\
+ \\
z \\
z \\
u\end{array}$ & 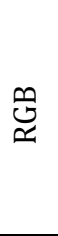 & 空 & 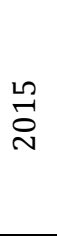 & 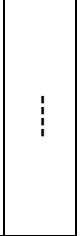 & & $\begin{array}{l}\text { The proposed system is validated on a large population-based } \\
\text { dataset over } 5378 \text { images, where it outperforms the state of the art } \\
\text { by extracting with respect to clinical grading a mean absolute error } \\
(\varepsilon) \text { of } 0.304 \text {, a } 70.7 \% \text { exact integral agreement ratio (R0), an } 88.4 \% \\
\text { decimal grading error } \leq 0.5 \text { (Re0.5), and a } 99.0 \% \text { decimal grading } \\
\text { error } \leq 1.0(\operatorname{Re} 1.0) \text {. }\end{array}$ \\
\hline$\underset{J}{\stackrel{\Xi}{U}}$ & 乙 & $\underset{\bar{x}}{\vec{x}}$ & $\underset{\Xi}{\stackrel{\Xi}{\Xi}}$ & \begin{tabular}{l}
0 \\
$\stackrel{\sim}{\circ}$ \\
\multirow{N}{*}{}
\end{tabular} & 궁 & & $\begin{array}{l}\text { Results on a dataset of X-ray images and KL grades from the } \\
\text { Osteoarthritis Initiative (OAI) show a sizable improvement over the } \\
\text { current state-of-the-art. }\end{array}$ \\
\hline
\end{tabular}


International Journal of Trend in Scientific Research and Development (IJTSRD) @ www.ijtsrd.com eISSN: 2456-6470

\begin{tabular}{|c|c|c|c|c|c|c|}
\hline 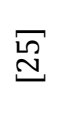 & Z & 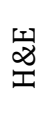 & 尊 & $\begin{array}{l}0 \\
\stackrel{1}{0} \\
\stackrel{N}{N}\end{array}$ & 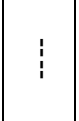 & This paper based on the Thyroid based machine learning. \\
\hline 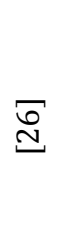 & 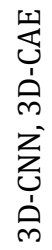 & $\widetilde{z}$ & 湑 & $\begin{array}{l}\underset{\sim}{\sim} \\
\stackrel{\sim}{N}\end{array}$ & 妾 & $\begin{array}{l}\text { Experiments on the ADNI MRI dataset with no skull-stripping } \\
\text { preprocessing have shown 3D-CNN outperforms several } \\
\text { conventional classifiers by accuracy and robustness. Abilities of the } \\
\text { 3D-CNN to generalize the features learnt and adapt to other domains } \\
\text { have been validated on the CAD Dementia dataset. }\end{array}$ \\
\hline$\stackrel{\widehat{N}}{ }$ & $\sum_{\substack{Z \\
\sum}}$ & 怘 & $\frac{\Xi}{\tilde{n}}$ & $\begin{array}{l}0 \\
\stackrel{1}{\circ} \\
\stackrel{N}{*}\end{array}$ & 蒫 & $\begin{array}{l}\text { The entire network is fine-tuned in a fully learned end-to-end } \\
\text { optimization with auxiliary loss functions. In this paper show how } \\
\text { proposed novel multi-tract network yields higher classification } \\
\text { accuracy, outperforming state-of-the-art multi-scale approaches } \\
\text { when compared over a public skin lesion dataset. }\end{array}$ \\
\hline 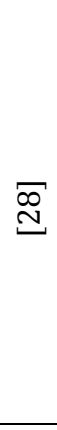 & z & 怘 & 空 & $\begin{array}{l}0 \\
\stackrel{\sim}{0} \\
\sim\end{array}$ & 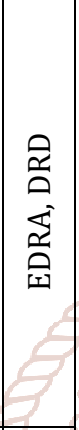 & $\begin{array}{l}\text { In this report investigate how different transfer learning schemes } \\
\text { influence automated melanoma classification results. This paper } \\
\text { evaluated transfer learning from a general-context dataset } \\
\text { (ImageNet) and from a specific-domain dataset (diabetic } \\
\text { retinopathy). Authors investigated if sequential transfer steps } \\
\text { improve the final classification result. This paper show results } \\
\text { consistent with the literature regarding training a DNN from scratch } \\
\text { and differences between doing fine-tuning or not. Author conclude } \\
\text { that general findings of deep learning state-of-the-art are also } \\
\text { applicable for automated melanoma screening literature, thus } \\
\text { guiding future research }\end{array}$ \\
\hline$\stackrel{\sigma}{\mathrm{d}}$ & $\sum_{\substack{Z \\
\sum}}^{1}$ & 它 & 占 & $\begin{array}{l}8 \\
0 \\
\stackrel{0}{0}\end{array}$ & 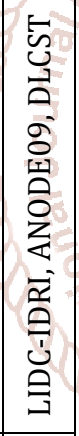 & $\begin{array}{l}\text { The proposed architecture comprises multiple streams of 2-D } \\
\text { ConvNets, for which the outputs are combined using a dedicated } \\
\text { fusion method to get the final classification. Data augmentation and } \\
\text { dropout are applied to avoid overfitting. On } 888 \text { scans of the publicly } \\
\text { available LIDCIDRI dataset, our method reaches high detection } \\
\text { sensitivities of } 85.4 \% \text { and } 90.1 \% \text { at } 1 \text { and } 4 \text { false positives per scan, } \\
\text { respectively. An additional evaluation on independent datasets from } \\
\text { the ANODE09 challenge and DLCST is performed. This paper showed } \\
\text { that the proposed multi-view ConvNets is highly suited to be used } \\
\text { for false positive reduction of a CAD system. }\end{array}$ \\
\hline 호 & $\begin{array}{l}\text { Ż } \\
\text { @ि } \\
\text { m }\end{array}$ & 它 & $\stackrel{00}{\Xi}$ & $\stackrel{\infty}{\underset{\sim}{\sigma}}$ & 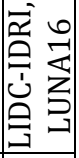 & $\begin{array}{l}\text { In this paper Extensive experimental results demonstrate that Deep } \\
\text { Lung has performance comparable to experienced doctors both for } \\
\text { the nodule-level and patient-level diagnosis on the LIDC-IDRI } \\
\text { dataset. }\end{array}$ \\
\hline $\overrightarrow{\vec{m}}$ & 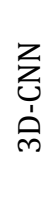 & $\widetilde{\Omega}$ & 旅 & \begin{tabular}{l}
$\sigma$ \\
$\stackrel{\sim}{\circ}$ \\
\multirow{N}{*}{}
\end{tabular} & 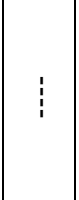 & $\begin{array}{l}\text { Experimental results demonstrate that the methods can achieve an } \\
\text { accuracy as high as } 89.9 \% \text { this paper also find that the learned } \\
\text { features from fMRI and DTI play more important roles in accurately } \\
\text { predicting the OS time, which provides valuable insights into } \\
\text { functional neuro-oncological applications. }\end{array}$ \\
\hline$\underset{\tilde{m}}{\widetilde{U}}$ & 空 & $\begin{array}{l}\Xi \\
\text { v } \\
\end{array}$ & 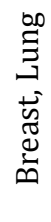 & $\begin{array}{l}\underset{\sim}{\sim} \\
\stackrel{\sim}{N}\end{array}$ & 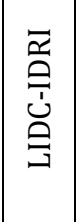 & $\begin{array}{l}\text { The experimental results show the significant performance boost by } \\
\text { the SDAE-based CADx algorithm over the two conventional methods, } \\
\text { suggesting that deep learning techniques can potentially change the } \\
\text { design paradigm of the CADx systems without the need of explicit } \\
\text { design and selection of problem-oriented features. }\end{array}$ \\
\hline$\stackrel{m}{m}$ & 亗 & $\stackrel{\Xi}{\Sigma}$ & 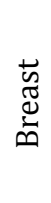 & 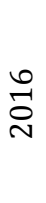 & 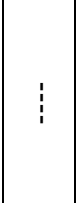 & $\begin{array}{l}\text { The state-of-the-art results show that the learned breast density } \\
\text { scores have a very strong positive relationship with manual ones, } \\
\text { and that the learned texture scores are predictive of breast cancer. } \\
\text { The model is easy to apply and generalizes too many other } \\
\text { segmentation and scoring problems. }\end{array}$ \\
\hline
\end{tabular}


International Journal of Trend in Scientific Research and Development (IJTSRD) @ www.ijtsrd.com eISSN: 2456-6470

\begin{tabular}{|c|c|c|c|c|c|c|c|}
\hline ঙ & $\sum_{\substack{Z\\
}}$ & $\stackrel{\Xi}{\Sigma}$ & 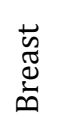 & 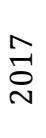 & 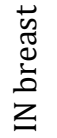 & & $\begin{array}{l}\text { Experimental results on the IN breast dataset demonstrate the } \\
\text { robustness of proposed networks compared to previous work using } \\
\text { segmentation and detection annotations. }\end{array}$ \\
\hline $\begin{array}{l}\text { ஸू } \\
\text { m }\end{array}$ & 己 & $\overrightarrow{\underline{\Sigma}}$ & 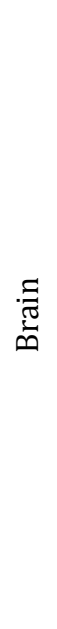 & 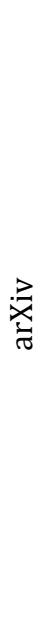 & 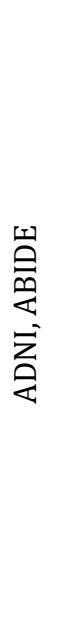 & & $\begin{array}{l}\text { This paper, introduce the novel concept of Graph Convolutional } \\
\text { Networks (GCN) for brain analysis in populations, combining } \\
\text { imaging and non-imaging data. This paper represent populations as } \\
\text { a sparse graph where its vertices are associated with image-based } \\
\text { feature vectors and the edges encode phenotypic information. This } \\
\text { structure was used to train a GCN model on partially labelled graphs, } \\
\text { aiming to infer the classes of unlabeled nodes from the node features } \\
\text { and pairwise associations between subjects. The Paper demonstrate } \\
\text { the potential of the method on the challenging ADNI and ABIDE } \\
\text { databases, as a proof of concept of the benefit from integrating } \\
\text { contextual information in classification tasks. This has a clear impact } \\
\text { on the quality of the predictions, leading to 69.5\% accuracy for } \\
\text { ABIDE (outperforming the current state of the art of } 66.8 \% \text { ) and } \\
77 \% \text { for ADNI for prediction of MCI conversion, significantly } \\
\text { outperforming standard linear classifiers where only individual } \\
\text { features are considered. }\end{array}$ \\
\hline $\begin{array}{l}\overline{6} \\
m\end{array}$ & z & 怘 & $\frac{\Xi}{\tilde{y}}$ & $\stackrel{\sqrt{2}}{\stackrel{D}{v}}$ & $\frac{1}{6}$ & & $\begin{array}{l}\text { This paper demonstrate classification of skin lesions using a single } \\
\text { CNN, trained end-to-end from images directly, using only pixels } \\
\text { and disease labels as inputs. Authors train a CNN using a dataset of } \\
129,450 \text { clinical images - two orders of magnitude larger than } \\
\text { previous datasets - consisting of } 2,032 \text { different diseases. It test } \\
\text { its performance against } 21 \text { board-certified dermatologists on } \\
\text { biopsy-proven clinical images with two critical binary } \\
\text { classification use cases: keratinocyte carcinomas versus benign } \\
\text { seborrheic keratosis; and malignant melanomas versus benign } \\
\text { nevi. The first case represents the identification of the most } \\
\text { common cancers, the second represents the identification of the } \\
\text { deadliest skin cancer. The CNN achieves performance on par with } \\
\text { all tested experts across both tasks, demonstrating an artificial } \\
\text { intelligence capable of classifying skin cancer with a level of } \\
\text { competence comparable to dermatologists. Outfitted with deep } \\
\text { neural networks, mobile devices can potentially extend the reach } \\
\text { of dermatologists outside of the clinic. }\end{array}$ \\
\hline$\widehat{\widehat{m}}$ & $\begin{array}{l}Z \\
己 \\
+ \\
\text { Z } \\
\text { Z }\end{array}$ & $\overrightarrow{\underline{\Sigma}}$ & 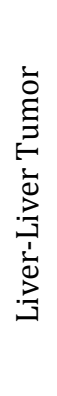 & 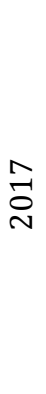 & 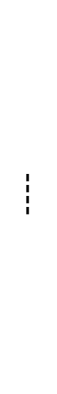 & & $\begin{array}{l}\text { In this method the framework achieves an end-to-end accuracy of } \\
65 \% \text { with a Dice score for the automatic lesion segmentation of } 69 \% \\
\text { and an accuracy of } 68 \% \text { for tumor malignancy classification based on } \\
\text { expert annotations. Authors compared the Survival Net to classical } \\
\text { handcrafted features such as Histogram and Horlicks and show } \\
\text { experimentally that Survival Net outperforms the handcrafted } \\
\text { features in HCC malignancy classification. End-to-end assessment of } \\
\text { tumor malignancy based on our proposed fully automatic framework } \\
\text { corresponds to assessment based on expert annotations with high } \\
\text { significance }(p>0.95) \text {. }\end{array}$ \\
\hline $\begin{array}{l}\infty \\
\stackrel{\infty}{m} \\
\underline{4} .\end{array}$ & $\stackrel{\mathrm{g}}{\mathrm{B}}$ & 它 & 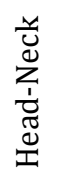 & 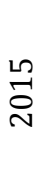 & & 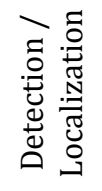 & $\begin{array}{l}\text { The proposed method has been quantitatively valuated for carotid } \\
\text { artery bifurcation election on a head- neck CT dataset from } 455 \\
\text { patients. Compared to the state-of-the-art, the mean error is reduced } \\
\text { by more than half, from } 5.97 \mathrm{~mm} \text { to } 2.64 \mathrm{~mm} \text {, with a detection speed } \\
\text { of less than } 1 \mathrm{~s} \text { /volume. }\end{array}$ \\
\hline
\end{tabular}




\begin{tabular}{|c|c|c|c|c|c|c|}
\hline $\bar{m}$ & Z & $\mathscr{s}$ & 苞 & $\stackrel{\text { L }}{\stackrel{7}{\circ}}$ & & $\begin{array}{l}\text { Authors Propose a transfer learning strategy, which transfers the } \\
\text { knowledge in the low layers of a base CNN trained from a large } \\
\text { database of natural images to our task-specific CNN. Extensive } \\
\text { experiments demonstrate that our approach outperforms the state- } \\
\text { of-the-art method for the FASP localization as well as the CNN only } \\
\text { trained on the limited US training samples. The proposed approach } \\
\text { can be easily extended to other similar medical image computing } \\
\text { problems, which often suffer from the insufficient training samples } \\
\text { when exploiting the deep CNN to represent high-level features. }\end{array}$ \\
\hline ஓ & $\begin{array}{l}Z \\
\text { Zu } \\
\text { ஸे } \\
\text { ปn }\end{array}$ & $\overrightarrow{\underline{\prime}}$ & 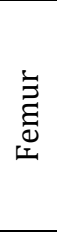 & $\stackrel{\text { L }}{\stackrel{7}{\circ}}$ & ষ্ঠ & $\begin{array}{l}\text { This paper used the narrow-band graph cut optimization to achieve } \\
\text { the 3D segmentation of femur surface. Finally, the anatomical } \\
\text { landmarks are located on the femur according to the geometric cues } \\
\text { of surface mesh. Experiments demonstrate that the proposed } \\
\text { method is effective, efficient, and reliable to segment femur and } \\
\text { locate the anatomical landmarks }\end{array}$ \\
\hline$\underset{\Xi}{\Xi}$ & 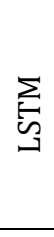 & $\mathscr{\rho}$ & 苞 & $\stackrel{\text { Ln }}{\stackrel{7}{\circ}}$ & & $\begin{array}{l}\text { This paper propose a joint learning framework with knowledge } \\
\text { transfer across multi-tasks to address the insufficiency issue of } \\
\text { limited training data. Extensive experiments on different US } \\
\text { standard planes with hundreds of videos corroborate that method } \\
\text { can achieve promising results, which outperform state-of-the-art } \\
\text { methods. }\end{array}$ \\
\hline$\underset{\mathcal{Z}}{\stackrel{乛}{Z}}$ & Z & $\frac{\vec{\pi}}{\grave{x}} \stackrel{\vec{x}}{\Sigma}$ & $\begin{array}{l}\text { D } \\
\underset{I}{I}\end{array}$ & $\begin{array}{l}0 \\
\stackrel{1}{\circ} \\
\text { N }\end{array}$ & 岕 & $\begin{array}{l}\text { This paper describe a machine learning method based on the CNN } \\
\text { applied on X-ray, MRI. }\end{array}$ \\
\hline$\stackrel{\Xi}{\Xi}$ & Z & 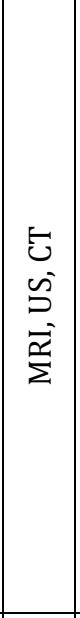 & ' & & 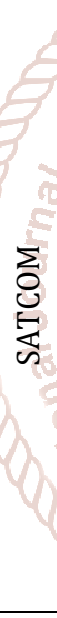 & $\begin{array}{l}\text { This paper describe a new learning method following a } \\
\text { fundamentally different paradigm by simultaneously modeling both } \\
\text { the object appearance and the parameter search strategy as a unified } \\
\text { behavioral task for an artificial agent. The method combines the } \\
\text { advantages of behavior learning achieved through reinforcement } \\
\text { learning with effective hierarchical feature extraction achieved } \\
\text { through deep learning. The paper show that given only a sequence of } \\
\text { annotated images, the agent can automatically and strategically learn } \\
\text { optimal paths that converge to the sought anatomical landmark } \\
\text { location as opposed to exhaustively scanning the entire solution } \\
\text { space. The method significantly outperforms state-of-the art } \\
\text { machine learning and deep learning approaches both in terms of } \\
\text { accuracy and speed on 2D magnetic resonance images, 2D } \\
\text { ultrasound and 3D CT images, achieving average detection errors of } \\
\text { 1-2 pixels, while also recognizing the absence of an object from the } \\
\text { image. }\end{array}$ \\
\hline$\underset{\Xi}{\Psi}$ & $\underbrace{Z}_{I}$ & $\mathscr{\rho}$ & 䒿 & $\stackrel{\bullet}{\stackrel{0}{\circ}}$ & & $\begin{array}{l}\text { In this paper, considered a fully automated system based on } \\
\text { convolutional neural networks which can detect twelve standard } \\
\text { scan planes as defined by the UK fetal abnormality screening } \\
\text { programme. The network design allows real-time inference and can } \\
\text { be naturally extended to provide an approximate localization of the } \\
\text { fetal anatomy in the image. Such a framework can be used to } \\
\text { automate or assist with scan plane selection, or for the retrospective } \\
\text { retrieval of scan planes from recorded videos. The method is } \\
\text { evaluated on a large database of } 1003 \text { volunteer mid-pregnancy } \\
\text { scans. The paper show that standard planes acquired in a clinical } \\
\text { scenario are robustly detected with a precision and recall of } 69 \% \\
\text { and } 80 \% \text {, which is superior to the current state-of-the-art. } \\
\text { Furthermore, paper show that it can retrospectively retrieve correct } \\
\text { scan planes with an accuracy of } 71 \% \text { for cardiac views and } 81 \% \text { for } \\
\text { non-cardiac views. }\end{array}$ \\
\hline
\end{tabular}




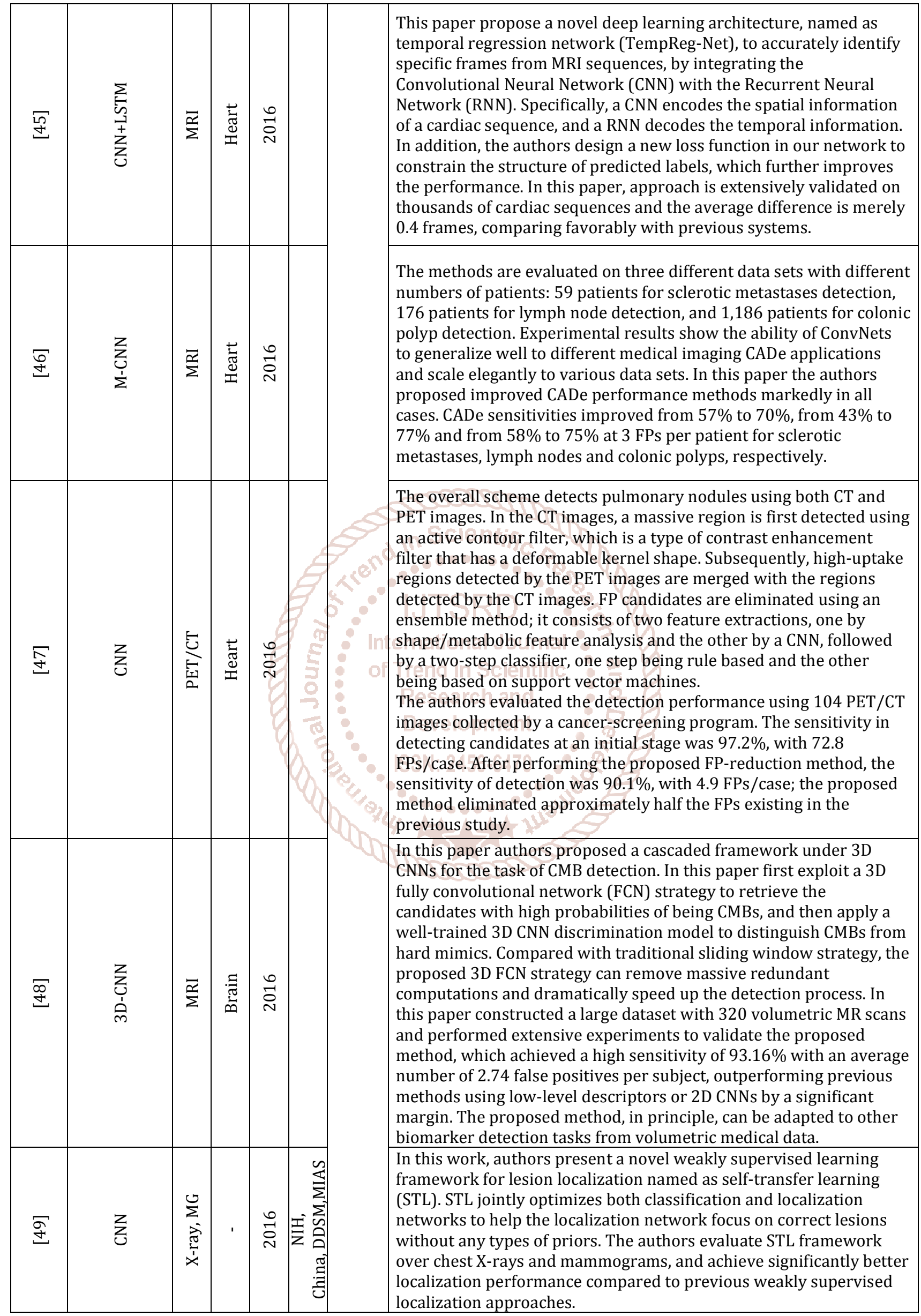




\begin{tabular}{|c|c|c|c|c|c|c|c|}
\hline $\begin{array}{l}\text { 오 } \\
\text { 는 }\end{array}$ & 忌 & 品 & 空 & $\begin{array}{l}0 \\
\stackrel{1}{0} \\
\sim\end{array}$ & 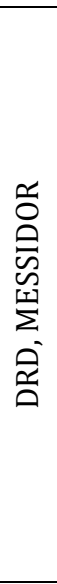 & & $\begin{array}{l}\text { In this paper, authors proposed a method to improve and speed-up } \\
\text { the CNN training for medical image analysis tasks by dynamically } \\
\text { selecting misclassified negative samples during training. Training } \\
\text { samples are heuristically sampled based on classification by the } \\
\text { current status of the CNN. Weights are assigned to the training } \\
\text { samples and informative samples are more likely to be included in } \\
\text { the next CNN training iteration. Authors evaluated and compared } \\
\text { our proposed method by training a CNN with (SeS) and without } \\
\text { (NSeS) the selective sampling method. The paper focus on the } \\
\text { detection of hemorrhages in color fundus images. A decreased } \\
\text { training time from } 170 \text { epochs to } 60 \text { epochs with an increased } \\
\text { performance-on par with two human experts-was achieved with } \\
\text { areas under the receiver operating characteristics curve of } 0.894 \text { and } \\
0.972 \text { on two data sets. The SeS CNN statistically outperformed the } \\
\text { NSeS CNN on an independent test set. }\end{array}$ \\
\hline 금 & 芯 & ' & ' & 공 & & & $\begin{array}{l}\text { In this paper authors proposed AnoGAN, a deep convolutional } \\
\text { generative adversarial network to learn a manifold of normal } \\
\text { anatomical variability, accompanying a novel anomaly scoring } \\
\text { scheme based on the mapping from image space to a latent space. } \\
\text { Applied to new data, the model labels anomalies, and scores image } \\
\text { patches indicating their fit into the learned distribution. Results on } \\
\text { optical coherence tomography images of the retina demonstrate that } \\
\text { the approach correctly identifies anomalous images, such as images } \\
\text { containing retinal fluid or hyper reflective foci. }\end{array}$ \\
\hline ํㅡ & Z & $\underset{\frac{\pi}{x}}{\grave{x}}$ & 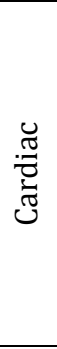 & $\begin{array}{l}0 \\
\stackrel{0}{0} \\
\end{array}$ & 5 & & $\begin{array}{l}\text { The authors proposed two novel methods for (i) fully automatic } \\
\text { electrophysiology catheter electrode detection in interventional X- } \\
\text { ray images and (ii) single-view depth estimation of such electrodes } \\
\text { based on convolutional neural networks. For (i), experiments on } 24 \\
\text { different fluoroscopy sequences ( } 1650 \mathrm{X} \text {-ray images) yielded a } \\
\text { detection rate }>99 \% \text {. In an experiments on (ii) depth prediction } \\
\text { using } 20 \text { images with depth information available revealed that we } \\
\text { are able to estimate the depth of catheter tips in the lateral view with } \\
\text { a remarkable mean error of } 6.08 \pm 4.666 .08 \pm 4.66 \mathrm{~mm} \text {. }\end{array}$ \\
\hline m & $\begin{array}{l}Z \\
\text { Ż } \\
\text { மे }\end{array}$ & 七 & $\stackrel{\infty}{\Xi}$ & 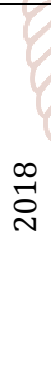 & 莫 & & $\begin{array}{l}\text { The nodule classification subnetwork was validated on a public } \\
\text { dataset from LIDC-IDRI, on which it achieved better performance } \\
\text { than state-of-the-art approaches and surpassed the performance of } \\
\text { experienced doctors based on image modality. Within the Deep Lung } \\
\text { system, candidate nodules are detected first by the nodule detection } \\
\text { subnetwork, and nodule diagnosis is conducted by the classification } \\
\text { subnetwork. Extensive experimental results demonstrate that Deep } \\
\text { Lung has performance comparable to experienced doctors both for } \\
\text { the nodule-level and patient-level diagnosis on the LIDC-IDRI } \\
\text { dataset. }\end{array}$ \\
\hline 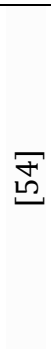 & 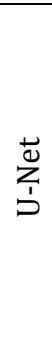 & ' & ' & $\stackrel{\text { 음 }}{\stackrel{0}{N}}$ & & 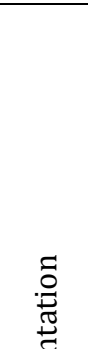 & $\begin{array}{l}\text { The authors show that such a network can be trained end-to-end } \\
\text { from very few images and outperforms the prior best method (a } \\
\text { sliding-window convolutional network) on the ISBI challenge for } \\
\text { segmentation of neuronal structures in electron microscopic stacks. } \\
\text { Using the same network trained on transmitted light microscopy } \\
\text { images (phase contrast and DIC) we won the ISBI cell tracking } \\
\text { challenge } 2015 \text { in these categories by a large margin. Moreover, the } \\
\text { network is fast. Segmentation of a 512x512 image takes less than a } \\
\text { second on a recent GPU. }\end{array}$ \\
\hline $\begin{array}{l}\text { 노 } \\
\text { 은 }\end{array}$ & Z & $\overrightarrow{\underline{\prime}}$ & 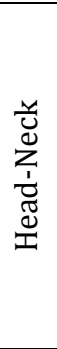 & $\begin{array}{l}0 \\
\stackrel{\circ}{\circ} \\
\text { }\end{array}$ & & 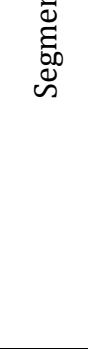 & $\begin{array}{l}\text { In this paper authors presents a pipeline is extensively evaluated on } \\
\text { three challenging tasks of lesion segmentation in multi-channel MRI } \\
\text { patient data with traumatic brain injuries, brain tumors, and } \\
\text { ischemic stroke. Authors improve on the state-of-the art for all three } \\
\text { applications, with top ranking performance on the public } \\
\text { benchmarks BRATS } 2015 \text { and ISLES } 2015 \text {. The method is } \\
\text { computationally efficient, which allows its adoption in a variety of } \\
\text { research and clinical settings. The source code of our } \\
\text { implementation is made publicly available. }\end{array}$ \\
\hline
\end{tabular}




\begin{tabular}{|c|c|c|c|c|c|c|c|}
\hline \multirow[t]{2}{*}{ అִ } & Z & 包 & 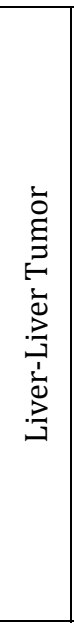 & $\begin{array}{l}\stackrel{0}{ } \\
\stackrel{\sim}{N}\end{array}$ & & & $\begin{array}{l}\text { This paper presents a method to automatically segment liver and } \\
\text { lesions in CT abdomen images using cascaded fully convolutional } \\
\text { neural networks (CFCNs) and dense 3D conditional random fields } \\
\text { (CRFs). Authors train and cascade two FCNs for a combined } \\
\text { segmentation of the liver and its lesions. In the first step, we train a } \\
\text { FCN to segment the liver as ROI input for a second FCN. The second } \\
\text { FCN solely segments lesions from the predicted liver ROIs of step } 1 . \\
\text { Refine the segmentations of the CFCN using a dense 3D CRF that } \\
\text { accounts for both spatial coherence and appearance. CFCN models } \\
\text { were trained in a } 2 \text {-fold cross-validation on the abdominal CT } \\
\text { dataset 3DIRCAD comprising } 15 \text { hepatic tumor volumes. Our results } \\
\text { show that CFCN-based semantic liver and lesion segmentation } \\
\text { achieves Dice scores over } 94 \% \text { for liver with computation times } \\
\text { below 100s per volume. This paper experimentally demonstrate the } \\
\text { robustness of the proposed method as a decision support system } \\
\text { with a high accuracy and speed for usage in daily clinical routine. }\end{array}$ \\
\hline & 它忌 & $\widetilde{\Sigma}$ & $\cong 0$ & 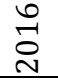 & ! & & $\begin{array}{l}\text { Model-Based Segmentation of Vertebral Bodies from MR Images } \\
\text { with 3D CNNs }\end{array}$ \\
\hline ํㅡㄴ & Z & 包 & 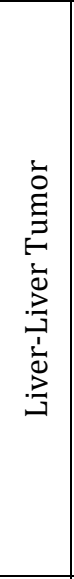 & $\stackrel{\sim}{\circ}$ & & & $\begin{array}{l}\text { This paper presents a method to automatically segment liver and } \\
\text { lesions in CT and MRI abdomen images using cascaded fully } \\
\text { convolutional neural networks (CFCNs) enabling the segmentation } \\
\text { of a large-scale medical trial or quantitative image analysis. Authors } \\
\text { train and cascade two FCNs for a combined segmentation of the liver } \\
\text { and its lesions. In the first step, we train a FCN to segment the liver } \\
\text { as ROI input for a second FCN. The second FCN solely segments } \\
\text { lesions within the predicted liver ROIs of step } 1 \text {. CFCN models were } \\
\text { trained on an abdominal CT dataset comprising } 100 \text { hepatic tumor } \\
\text { volumes. Validations on further datasets show that CFCN-based } \\
\text { semantic liver and lesion segmentation achieves Dice scores over } \\
\text { 94\% for liver with computation times below 100s per volume. } \\
\text { Authors further experimentally demonstrate the robustness of the } \\
\text { proposed method on an } 38 \text { MRI liver tumor volumes and the public } \\
\text { 3DIRCAD dataset. tific }\end{array}$ \\
\hline$\underset{\infty}{\infty}$ & Z & $\widetilde{\Sigma}$ & 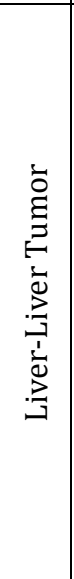 & $\stackrel{\curvearrowright}{\circ}$ & $\frac{9}{6}$ & 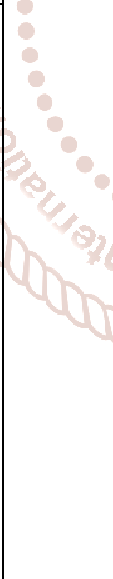 & $\begin{array}{l}\text { A 3D neural network (Survival Net) then predicts the HCC lesions' } \\
\text { malignancy from the HCC tumor segmentation. We formulate this } \\
\text { task as a classification problem with classes being "low risk" and } \\
\text { "high risk" represented by longer or shorter survival times than the } \\
\text { median survival. We evaluated our method on DWI of } 31 \text { HCC } \\
\text { patients. Our proposed framework achieves an end-to-end accuracy } \\
\text { of } 65 \% \text { with a Dice score for the automatic lesion segmentation of } \\
69 \% \text { and an accuracy of } 68 \% \text { for tumor malignancy classification } \\
\text { based on expert annotations. Authors compared the Survival Net to } \\
\text { classical handcrafted features such as Histogram and Horlick and } \\
\text { show experimentally that Survival Net outperforms the handcrafted } \\
\text { features in HCC malignancy classification. End-to-end assessment of } \\
\text { tumor malignancy based on our proposed fully automatic framework } \\
\text { corresponds to assessment based on expert annotations with high } \\
\text { significance (p>0.95). }\end{array}$ \\
\hline a & $\begin{array}{l}Z \\
\text { ż } \\
\text { mे }\end{array}$ & 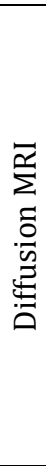 & 节 & $\begin{array}{l}0 \\
\stackrel{\sim}{0} \\
\sim\end{array}$ & & & $\begin{array}{l}\text { The authors demonstrate how deep learning, a group of algorithms } \\
\text { based on recent advances in the field of artificial neural networks, } \\
\text { can be applied to reduce diffusion MRI data processing to a single } \\
\text { optimized step. This modification allows obtaining scalar measures } \\
\text { from advanced models at twelve-fold reduced scan time and } \\
\text { detecting abnormalities without using diffusion models. We set a } \\
\text { new state of the art by estimating diffusion kurtosis measures from } \\
\text { only } 12 \text { data points and neurite orientation dispersion and density } \\
\text { measures from only } 8 \text { data points. This allows unprecedentedly fast } \\
\text { and robust protocols facilitating clinical routine and demonstrates } \\
\text { how classical data processing can be streamlined by means of deep } \\
\text { learning. }\end{array}$ \\
\hline ఠ。 & 㤂 & $\stackrel{\Xi}{\Sigma}$ & 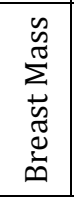 & $\stackrel{\infty}{\underset{\sim}{\sim}}$ & & & $\begin{array}{l}\text { Multi-scale FCN is employed to improve the segmentation } \\
\text { performance. Experimental results on two public datasets, IN breast } \\
\text { and DDSM-BCRP, demonstrate that in this paper end-to-end network } \\
\text { achieves better performance than state-of-the-art approaches. IN } \\
\text { breast, DDSM-BCRP }\end{array}$ \\
\hline
\end{tabular}


years. Since all the above mentioned methods are very important and use full in medical field and PR. The value potential is everywhere, even in industries that have been slow to digitize. These technologies could generate productivity gains and an improved quality of life-along with job losses and other disruptions. Previous research found that 45 percent of work activities could potentially be automated by currently demonstrated technologies; machine learning can be an enabling technology for the automation of 80 percent of those activities. Breakthroughs in natural language processing could expand that impact even further.

\section{References}

[1] Hsinchun Chen, "Machine Learning for Information Retrieval: Neural Networks, Symbolic Learning, and Genetic Algorithms", available at http://ai.bpa.arizona.edu/papers/mlir93/mlir93.html \#318.

[2] Peter Flach and Nada Lavrac,"Rule Induction" www.cs.bris.ac.uk/Teaching/Resources/COMSM0301/ materials/RuleInductionSection.pdf.

[3] Junfei Qiu, Qihui Wu, Guoru Ding, Yuhua Xu and Shuo Feng, "A survey of machine learning for big data processing", Qiuet al. EURASIP Journal on Advances in Signal Processing, pp.1-16 , 2016:67, 2016.

[4] Soumava Roy, Samitha Herath, Richard Nock, and Fatih Porikli, "Machines that Learn with Limited or No Supervision: A Survey on Deep Learning Based Techniques", 2017.

[5] Geoffrey Hinton, Oriol Vinyals, and Jeff Dean, "Distilling the knowledge in a neural network", arXiv preprint arXiv: 1503.02531, 2015.

[6] Zadeh, L.A. (1988), "Fuzzy Logic", IEEE Computer, pp. 83-89, 1988.

[7] Srinivasan Arunachalam, Ronald de Wolf, "A Survey of Quantum Learning Theory" siact arxiv.

[8] Cristian Romero Garcia, "Quantum Machine Learning" Ph.D. Thesis -Department of Physical Chemistry Faculty of Science and Technology University of the Basque Country UPV/EHU Leioa, September, 2016.

[9] http://bair.berkeley.edu/blog/2017/07/18/learningto-learn/

[10] C. Watkins and P. Dayan, "Q-learning", Machine learning, vol. 8, no. 3, pp. 279-292, 1992.

[11] M.S. Mahdavinejad, M. Rezvan, M. Barekatain, P. Adibi, P. Barnaghi, A.P. Sheth, "Machine learning for Internet of Things data analysis: A survey", Digital Communications and Networks, doi: 10.1016/j.dcan.2017.10.002,pp.1-57, 2017.

[12] A. Thomaz and C. Breazeal, "Teachable robots: Understanding human teaching behavior to build more effective robot learners", Artificial Intelligence, vol. 172, no. 6-7, pp. 716-737, 2008.

[13] Hebb's book, "The Organization of Behavior : A Neuropsychological Theory", New York: Willey (1949, p. 62)

[14] Simon Hykin, "Neural Network: A comprehensive Foundation", Prentice Hall.

[15] Adam Gibson et al.,(2018)https://deeplearning4j.org/convolutionalnet work.

[16] Techopedia.(2018),https://www.techopedia.com/defi nition/32834/recurrent-neural-network-rnn.
[17] DishashreeGupta.(2017),https://www.analyticsvidhya. com/blog/2017/12/introduction-to-recurrentneuralnetworks/

[18] Yan Xu ; Tao Mo ; Qiwei Feng; Peilin Zhong; Maode Lai ; Eric I-Chao Chang, "Deep learning of feature representation with multiple instance learning for medical image analysis", 2014 IEEE International Conference on Acoustics, Speech and Signal Processing (ICASSP), Electronic ISBN: 978-1-4799-2893-4

[19] Shadi Albarqouni*, Student Member, IEEE, Christoph Baur, Felix Achilles, Student Member, IEEE, Vasileios Belagiannis, Student Member, IEEE, Stefanie Demirci, and Nassir Navab, Member, IEEE, "AggNet: Deep Learning From Crowds for Mitosis Detection in Breast Cancer Histology Images", IEEE TRANSACTIONS ON MEDICAL IMAGING, VOL. 35, NO. 5, MAY 2016, Pages1313-1321

[20] Lin Yang, Yizhe Zhang, Jianxu Chen, Siyuan Zhang, Danny Z. Chen, "Suggestive Annotation: A Deep Active Learning Framework for Biomedical Image Segmentation", arXiv:1706.04737v1 [cs.CV] 15 Jun 2017,pages $1-8$

[21] Shen W., Zhou M., Yang F., Yang C., Tian J. (2015) Multiscale Convolutional Neural Networks for Lung Nodule Classification. In: Ourselin S., Alexander D., Westin CF., Cardoso M. (eds) Information Processing in Medical Imaging. IPMI 2015. Lecture Notes in Computer Science, vol 9123. Springer, Cham, Print ISBN978-3319-19991-7

[22] Adrien Payan, Giovanni Montana, "Predicting Alzheimer's disease: a neuroimaging study with 3D convolutional neural networks", Computer Vision and Pattern Recognition, arXiv,2015

[23] Xinting Gao, Stephen Lin, Tien Yin Wong, "Automatic Feature Learning to Grade Nuclear Cataracts Based on Deep Learning", Published 2014 in IEEE Transactions on Biomedical Engineering

[24] Joseph Antony, Kevin McGuinness, Noel E O'Connor, Kieran Moran, "Quantifying Radiographic Knee Osteoarthritis Severity using Deep Convolutional Neural Networks", arXiv:1609.02469v1 [cs.CV] 8 Sep 2016,pages 1-6

[25] Edward Kim, Edward Kim, Miguel Corte-Real, Miguel Corte-Real, Zubair Baloch, Zubair Baloch,, "A Deep Semantic Mobile Application for Thyroid Cytopathology", Proc. SPIE 9789, Medical Imaging 2016: PACS and Imaging Informatics: Next Generation and Innovations, 97890A (5 April 2016); doi: 10.1117/12.2216468; https://doi.org/10.1117/12.221 6468

[26] Ehsan Hosseini-Asl , Georgy Gimel'farb , Ayman El-Baz, for the Alzheimer's Disease Neuroimaging Initiative, “Alzheimer's Disease Diagnostics by a Deeply 
Supervised Adaptable 3D Convolutional Network", arXiv,2016.

[27] Jeremy Kawahara, and Ghassan Hamarneh, "Multiresolution-tract CNN with hybrid pretrained and skinlesion trained layers",MLMI,2016

[28] Afonso Menegola, Michel Fornaciali , Ramon Pires, Sandra Avila, Eduardo Valle, "Towards Automated Melanoma Screening: Exploring Transfer Learning Schemes",arXiv,2016

[29] Arnaud Arindra Adiyoso Setio*, Fran cesco Ciompi, Geer Litjens, Paul Gerke, Colin Jacobs, Sarah J. van Riel, Mathilde Marie Winkler Wille, Matiullah Naqibullah, Clara I. Sánchez, and Bram van Ginneken., "Pulmonary Nodule Detection in CT Images: False Positive Reduction Using Multi-View Convolutional Networks", March 2016, IEEE Transactions on Medical Imaging 35(5):1-1 DOI: 10.1109/TMI.2016.2536809

[30] Wentao Zhu, Chaochun Liu, Wei Fan, Xiaohui Xie, "DeepLung: Deep 3D Dual Path Nets for Automated Pulmonary Nodule Detection and Classification", IEEE WACV conference, 2018

[31] Nie D, Zhang H, Adeli E, Liu L, Shen D., "3D Deep Learning for Multi-modal Imaging-Guided Survival Time Prediction of Brain Tumor Patients", Med Image Comput Assist Interv. 2016 Oct; 9901:212-220. doi: 10.1007/978-3-319-46723-8_25. Epub 2016 Oct 2

[32] Jie-ZhiCheng, Dong Ni, Yi-HongChou, JingQin, ChuiMeiTiu, Yeun-ChungChang, Chiun-Sheng Huang, Dinggang Shen \& Chung-MingChen, "Computer-Aided Diagnosis with Deep Learning Architecture: Applications to Breast Lesions in US Images and Pulmonary Nodules in CT Scans

[33] Michiel Kallenberg, Kersten Petersen, Mads Nielsen, Andrew Y. Ng, Pengfei Diao, Christian Igel, Celine M. Vachon, Katharina Holland, Rikke Rass Winkel, Nico Karssemeijer, Martin Lillholm,"Unsupervised deep learning applied to breast density segmentation and mammographic risk scoring" IEEE Transactions on Medical Imaging ( Volume: 35, Issue: 5, May 2016 )

[34] Wentao Zhu,Qi Lou,Yeeleng Scott Van,g,Xiaohui Xie,, "Deep multi-instance networks with sparse label assignment for whole mammogram classification", International Conference on Medical Image Computing and Computer-Assisted Intervention,",MICCAI 2017: Medical Image Computing and Computer-Assisted Intervention MICCAI 2017 pp 603-611

[35] Spectral Graph Convolutions for Population-based Disease Prediction

[36] Andre Esteva, Brett Kuprel, Roberto A. Novoa, Justin Ko, Susan M. Swetter, Helen M. Blau \& Sebastian Thrun, "Dermatologist-level classification of skin cancer with deep neural networks", Nature volume542, pages115-118 (02 February 2017)

[37] Patrick Ferdinand Christ, Florian Ettlinger, Georgios Kaissis, Sebastian Schlecht, Freba Ahmaddy, Felix Grün, Alexander Valentinitsch, Seyed-Ahmad Ahmadi, Rickmer Braren, Bjoern Menze, "SurvivalNet: Predicting patient survival from diffusion weighted magnetic resonance images using cascaded fully convolutional and 3D convolutional neural networks", Computer Vision and Pattern Recognition, IEEE ISBI 2017

[38] Yefeng Zheng, David Liu, Bogdan Georgescu, Hien Nguyen, and Dorin Comaniciu, "3D Deep Learning for Efficient and Robust Landmark Detection in Volumetric Dat",MICCAI,2015

[39] Hao Chen; Dong Ni; Jing Qin; Shengli Li; Xin Yang; Tianfu Wang; Pheng Ann Heng "Standard Plane Localization in Fetal Ultrasound via Domain Transferred Deep Neural Networks", IEEE Journal of Biomedical and Health Informatics ( Volume: 19, Issue: 5, Sept. 2015 )Page(s): 1627 - 1636

[40] Dong Yang, Shaoting Zhang, Zhennan Yan , Chaowei Tan, Kang Li, Dimitris Metaxas, "Automated anatomical landmark detection ondistal femur surface using convolutional neural network", ISBI,2015

[41] Hao Chen,Qi Dou,Dong Ni,JieZhi Cheng,Jing Qin,,Shengli Li,Pheng-Ann Heng, "Automatic Fetal Ultrasound Standard Plane Detection Using Knowledge Transferred Recurrent Neural Networks", Lecture Notes in Computer Science book series (LNCS, volume 9349)

[42] Christian Payer, Darko Stern, Horst Bischof, and Martin Urschler, "Regressing Heatmaps for Multiple Landmark Localization using CNNs", MICCAI,2016

[43] Florin C. Ghesu, Bogdan Georgescu, Tommaso Mansi, Dominik Neumann,Joachim Hornegger, and Dorin Comaniciu, "An artificial agent for anatomical landmark detection in medical images",MICCAI,2016

[44] C.F. Baumgartner, K. Kamnitsas, J. Matthew, S. Smith, B. Kainz, and D. Rueckert, "Real-time Standard Scan Plane Detection and Localisation in Fetal Ultrasound using Fully Convolutional Neural Networks", MICCAI,2016

[45] Bin Kong, Yiqiang Zhan, Min Shin, Thomas Denny, Shaoting Zhang, "Recognizing end-diastole and endsystole frames via deep temporal regression network ",Lecture Notes in Computer Science book series (LNCS, volume 9902), MICCAI,2016

[46] Holger R. Roth, Le Lu, EEE, Jiamin Liu, Jianhua Yao, Ari Seff, Kevin Cherry, Lauren Kim, and Ronald M. Summers, "Improving Computer-Aided Detection Using Convolutional Neural Networks and Random View Aggregation Neural Networks", IEEE-TMI, 2016

[47] Atsushi Teramoto, Hiroshi Fujita, Osamu Yamamuro and Tsuneo Tamaki, "Automated detection of pulmonary nodules in PET/CT images: Ensemble falsepositive reduction using a convolutional neural network technique Neural Networks"

[48] Qi Dou, Hao Chen, Lequan Yu, Lei Zhao, Jing Qin Defeng Wang, Vincent CT Mok,Lin Shi and Pheng-Ann Heng, "Automatic Detection of Cerebral Microbleeds From MR Images via 3D Convolutional Neural Networks" IEEE-TMI,2016

[49] Sangheum Hwang, Hyo-Eun Kim, "Self-Transfer Learning for Fully Weakly Supervised Lesion Localization",MICCAI,2016 
[50] Mark J.J.P. van Grinsven ; Bram van Ginneken ; Carel B. Hoyng; Thomas Theelen ; Clara I. Sánchez, "Fast Convolutional Neural Network Training Using Selective Data Sampling: Application to Hemorrhage Detection in Color Fundus Images", IEEE Transactions on Medical Imaging,MICCAI,2016

[51] Thomas Schlegl, Philipp Seeböck, Sebastian M. Waldstein, Ursula Schmidt-Erfurth, Georg Langs,, "Unsupervised Anomaly Detection with Generative Adversarial Networks to Guide Marker Discovery" Computer Vision and Pattern Recognition, IPMI,2017

[52] Christoph Baur,Shadi Albarqouni,Stefanie Demirci,Nass ir Navab,Pascal Fallavollita," "CathNets: Detection and Single-View Depth Prediction of Catheter Electrodes", Lecture Notes in Computer Science book series (LNCS, volume 9805)MIAR 2016

[53] Wentao Zhu, Chaochun Liu, Wei Fan, Xiaohui Xie, "DeepLung: Deep 3D Dual Path Nets for Automated Pulmonary Nodule Detection and Classification", Computer Vision and Pattern Recognition, arXiv,2018

[54] Olaf Ronneberger, Philipp Fischer, Thomas Brox, " Unet: Convolutional networks for biomedical image segmentation',MICCAI,2015

[55] Konstantinos Kamnitsasa , Christian Lediga , Virginia F.J. Newcombe, Joanna P. Simpson, Andrew D. Kane, David K. Menon, Daniel Rueckert, Ben Glocker, 'Efficient multi-scale 3D CNN with fully connected CRF for accurate brain lesion segmentation",

[56] Patrick Ferdinand Christ, Mohamed Ezzeldin A. Elshaer, Florian Ettlinger, Sunil Tatavarty, Marc in Bickel, Patrick Bilic, Markus Rempfler, Marco Armbruster, Felix Hofmann, Melvin D'Anastasi, Wieland H. Sommer, Seyed-Ahmad Ahmadi, Bjoern H. Menze, "Automatic Liver and Lesion Segmentation in CT Using Cascaded Fully Convolutional Neural Networks and 3D Conditional Random Fields",

[57] Patrick Ferdinand Christ, Florian Ettlinger, Felix Grün, Mohamed Ezzeldin A. Elshaera, Jana Lipkova, Sebastian Schlecht, Freba Ahmaddy, Sunil Tatavarty, Marc Bickel, Patrick Bilic, Markus Rempfler, Felix Hofmann, Melvin D Anastasi, SeyedAhmad Ahmadi, Georgios Kaissis, Julian Holch,Wieland Sommer, Rickmer Braren, Volker Heinemann, Bjoern Menze, "Automatic Liver and Tumor Segmentation of CT and MRI Volumes using Cascaded Fully Convolutional Neural Networks",

[58] Patrick Ferdinand Christ, Florian Ettlinger, Georgios Kaissis, Sebastian Schlecht, Freba Ahmaddy, Felix
Grün, Alexander Valentinitsch, Seyed-Ahmad Ahmadi, Rickmer Braren, Bjoern Menze, "SurvivalNet: Predicting patient survival from diffusion weighted magnetic resonance images using cascaded fully convolutional and 3D convolutional neural networks

[59] Vladimir Golkov; Alexey Dosovitskiy; Jonathan I. Sperl; Marion I. Menzel; Michael Czisch; Philipp Sämann ; Thomas Brox, Daniel Cremers, " q-Space Deep Learning: Twelve-Fold Shorter and Model-Free Diffusion MRI",

[60] Wentao Zhu, Xiang Xiang, Trac D. Tran, Gregory D. Hager, Xiaohui Xie, "Adversarial Deep Structured Nets for Mass Segmentation from Mammograms

[61] Qi Dou, Hao Chen, Yueming Jin, Lequan Yu, Jing Qin, Pheng-Ann Heng, "3D Deeply Supervised Network for Automatic Liver Segmentation from CT Volumes",

[62] Konstantinos Kamnitsas, Christian Baumgartner Christian Ledig, Virginia Newcombe, Joanna Simpson, Andrew Kane, David Menon, Aditya Nori , Antonio Criminisi, Daniel Rueckert, and Ben Glocker, "Unsupervised domain adaptation in brain lesion segmentation with adversarial networks

[63] Rui Liao, Shun Miao, Pierre de Tournemire, Sasa Grbic, Ali Kamen, Tommaso Mansi, Dorin Comaniciu, "An Artificial Agent for Robust Image Registration.", AAAI Conference 2017,https://arxiv.org/pdf/1611.10336.pdf.

[64] Dong Yang, Shaoting Zhang, Zhennan Yan, Chaowei Tan , Kang Li, Dimitris Metaxas, "Automated anatomical landmark detection ondistal femur surface using convolutional neural network

[65] Vladimir Golkov; Alexey Dosovitskiy; Jonathan I. Sperl; Marion I. Menzel; Michael Czisch; Philipp Sämann ; Thomas Brox, Daniel Cremers, " q-Space Deep Learning: Twelve-Fold Shorter and Model-Free Diffusion MRI",

[66] Schlemper, J., Caballero, J., Hajnal, J. V., Price, A. N., \& Rueckert, D. (2018). A deep cascade of convolutional neural networks for dynamic MR image reconstruction. IEEE transactions on Medical Imaging, 37(2), 491-503.

[67] Morteza Mardani1, Enhao Gong1, Joseph Y. Cheng1,2, Shreyas Vasanawala, Lei Xing, John M. Pauly, " Deep Generative Adversarial Networks for Compressed Sensing Automates MRI

[68] https://www.ncbi.nlm.nih.gov/pubmed/28212054 